\title{
Reinventio e unanimidade. Impacto das políticas culturais e turísticas na Comunidade local de Santiago de Compostela
}

\author{
Reinventio and unanimity. Impact of cultural and tourist policies on the local \\ Community of Santiago de Compostela
}

\author{
CARLOS PAZOS-JUSTO \\ Universidade do Minho, Galabra \\ https://orcid.org/0000-0001-6172-3059 \\ carlospazos@ilch.uminho.pt \\ MARISA DEL RÍO \\ Universidade de Santiago de Compostela, Galabra \\ http://orcid.org/0000-0003-2712-1139 \\ marisa.delrio@usc.es \\ ROBERTO SAMARTIM \\ Universidade da Corunha, GET. Galabra \\ http://orcid.org/0000-0001-8704-2235 \\ roberto.samartin@udc.es
}

\section{RESUMO}

O texto aborda, em primeiro lugar, a síntese das principais linhas de força das políticas públicas relativas à cultura e ao turismo implementadas em Santiago de Compostela, com base em bibliografia específica e foco nos Caminhos de Santiago. Em segundo lugar, com recurso a um corpus construído a partir de inquéritos a comerciantes e outros membros da comunidade local compostelana, o artigo analisa os impactes destas políticas, a que chamaremos reinventio. Verificámos que esses impactes são percebidos pela comunidade local compostelana, em geral, em termos de unanimidade, mas com diferenças significativas entre os locais comerciantes e os não comerciantes.

Palavras-chave: Comunidade local. Políticas culturais. Turismo. Santiago de Compostela. Caminho de Santiago.

1 Este trabalho é resultado do projeto "Narrativas, usos e consumos de visitantes como aliados ou ameaças para o bem-estar da comunidade local: o caso de Santiago de Compostela" (Ref: FFI2017-88196-R), parcialmente subsidiado pelo Ministerio de Industria, Economía y Competitividad do Governo da Espanha no quadro do Programa Estatal de I+D+I Orientada a los Retos de la Sociedad (2018-2021). 


\begin{abstract}
The text addresses, firstly, the synthesis of the main parameters of public policies related to culture and tourism implemented in Santiago de Compostela, taking as a basis specific bibliography and focusing the Santiago Way. Secondly, the article analyzes the impact of these policies, which we will call reinventio, using a corpus of surveys of merchants and other members of the local community of Compostela. We have verified that these impacts are unanimously perceived by the local community in general, but with significant differences between local traders and non-traders.
\end{abstract}

Keywords: Local community. Cultural policies. Tourism. Santiago de Compostela. Way of Santiago.

\title{
1. INTRODUÇÃO
}

As políticas culturais, entendidas como um «programa de intervenções realizadas pelo Estado, instituições civis, entidades privadas ou grupos comunitários com o objetivo de satisfazer as necessidades culturais da população e promover o desenvolvimento de suas representações simbólicas» (Teixeira Coelho, 1997), não ficaram alheias aos processos de globalização e de mercantilização da cultura (Canelas Rubim, 2009). Neste quadro e progressivamente, a cultura tem sido equacionada como um fator relevante para o desenvolvimento das comunidades (UNESCO, 1998; Lopes, 2010; Rodriguez Morató, 2010; Paül i Agusti, 2013). Deste modo, globalização, mercantilização e desenvolvimento (conceitos ligados em regra ao alargamento social e geográfico do consumo e do crescimento económico, assentes como valores fortes na cultura gerada pelo modo de produção capitalista) podem ser perspetivados como elementos centrais das linhas de força a condicionar as políticas culturais das últimas décadas, em qualidade de desafios mas também como objetivos dos agentes envolvidos na articulação e execução dessas políticas. Paralelamente, a partir da década de 80 do século XX, em função do contínuo esvaziamento do papel do Estado no âmbito cultural, assistimos à emergência das cidades enquanto protagonistas da vida cultural, passando a funcionarem como objeto e sujeito privilegiado das políticas culturais (Rodríguez Morató, 2005; Bouzada, 2007 e 2008; Barbieri, 2014).

Estas políticas culturais estão condicionadas pelo turismo enquanto resultado igualmente das dinâmicas globalizadoras (Delgado, 2007; Pereiro \& Fernandes, 2018) e como fenómeno económico, social e cultural central nas sociedades ocidentais. Relativamente à comunidade local, por seu turno, há já uma literatura importante (cfr. infra) a analisar a relação entre aquela e as políticas culturais e/ou turísticas.

Centrada no «lado da procura», a Teoria do Intercâmbio Social (Blau, 1968) propõe a existência de uma relação entre a perceção e as atitudes da população local e a natureza do seu envolvimento com o turismo. Com esta perspetiva, o trabalho foca dois objetivos fundamentais: em primeiro lugar realiza um levantamento analítico da bibliografia específica sobre as políticas públicas para a cultura e o turismo implementadas em Santiago de Compostela, com foco nos Caminhos de Santiago. Num segundo momento, com recurso 
a um corpus construído a partir de inquéritos passados a habitantes e comerciantes de Santiago de Compostela, analisa a perceção dos impactes destas políticas na comunidade local. Na discussão subsequente testaremos a hipótese de que estas políticas públicas locais referidas à cultura e ao turismo (a que denominamos reinventio pelas razões adiantes expostas) são aceites com um alto grau de unanimidade pela população local, destacando um maior nível de aceitação naqueles sectores da comunidade com maior dependência económica do sector turístico. Finalmente, apresentaremos e discutiremos os resultados obtidos, as suas implicações e algumas propostas para futuras pesquisas.

\section{REVISÃO DA LITERATURA}

Como já foi referido, as políticas culturais estão condicionadas pelo turismo enquanto resultado (Delgado, 2007; Pereiro \& Fernandes, 2018) e como fenómeno económico, social e cultural central nas sociedades ocidentais². A municipalização das políticas culturais e a expansão do turismo como sector económico relevante têm contribuído para o surgimento de uma aliança entre cultura e turismo (Costa, 2005; Richards, 2014; Rodríguez Morató, 2010) com impactes em várias dimensões, amiúde em termos de turismo cultural $^{3}$ ou de turismo urbano (López Palomeque, 2015).

Exemplos nesta direção no âmbito peninsular supõem Bilbau (OMT, 2018: 81), Barcelona (Lopez Palomeque, 2015) ou, em Portugal, a «competência entre cidades» no âmbito cultural-turístico representado pelo Porto ou Guimarães (Remoaldo \& Cadima Ribeiro, 2017: 23); aqui, eventos culturais como as capitalidades europeias da cultura de 2001 e 2012, respetivamente, são colocadas como «oportunidade[s] preciosa[s] para reforçar a imagem da cidade, a nível nacional e internacional, como destino cultural» (Remoaldo \& Cadima Ribeiro, 2017: 39), assim como de ultrapassar, no caso vimaranense, a decadência que alguns sectores económicos tradicionais (indústria do têxtil, calçado ou cutelaria, por exemplo) tinham enfrentado durante as últimas décadas do século XX. Encontramos outras evidências de que as «Indústrias criativas e [o] turismo cultural transformaram-se em ativos estratégicos para as economias locais» no estudo encomendado

2 Em função das perspetivas da Organização Mundial do Turismo [OMT, 2017], este fenómeno está a caminho de se converter na primeira atividade económica mundial (Pereiro, 2009). Assim, em 2016 o número total mundial de turistas foi de 1.235 milhões, um $4 \%$ superior aos resultados de 2015 , e com um aumento continuado de sete anos consecutivos, também no âmbito das peregrinações, consideradas neste trabalho uma forma específica de turismo apesar de não haver um consenso claro neste sentido (Remoaldo \& Cadima Ribeiro, 2017; Pereiro \& Fernandes, 2018). Ainda segundo a OMT (2014), em 2014 entre 300 e 330 milhões de turistas visitavam destinos religiosos.

3 «Cultural tourism is a type of tourism activity in which the visitor's essential motivation is to learn, discover, experience and consume the tangible and intangible cultural attractions/products in a tourism destination. These attractions/products relate to a set of distinctive material, intellectual, spiritual and emotional features of a society that encompasses arts and architecture, historical and cultural heritage, culinary heritage, literature, music, creative industries and the living cultures with their life styles, value systems, beliefs and traditions» (OMT, 2018). 
em 2015 pela Confederação Internacional das Sociedades de Autores e Compositores de Portugal (EY, 2015: 20) ou no Plano Estratégico para o Desenvolvimento de Braga 20142026 (INVESTBRAGA, 2014), cidade esta que está a preparar a sua candidatura a Capital Europeia da Cultura em 2027, e em que a aliança entre turismo e cultura (e religião) é um dos vetores assinalados. No caso bracarense, aliás, destaca-se a promoção do turismo cultural e religioso em sintonia com a estratégia de planificação da entidade regional responsável (Turismo do Porto e Norte de Portugal, 2015), com Santiago de Compostela como modelo de desenvolvimento turístico para alguns autores (Pereira \& Peres, 2010).

$\mathrm{Na}$ Galiza, por seu lado, «encontramos durante el período comprendido entre 1990 y 2005, una política cultural muy vinculada a la política turística» (Lage, Losada \& Gómez, 2012: 123), só desvinculada de 2005 a 2009 durante o governo de coaligação entre PSdeG-BNG (progressistas federalistas e nacionalistas galegos de esquerda) ${ }^{4}$. Na tríade emergente cidade-cultura-turismo, a organização de (mega)eventos (capitalidade europeia da cultura, macroconcertos, etc.) ou a dotação de grandes equipamentos culturais (pense-se na Auditorio de Galicia em Compostela ou na Casa da Música do Porto) configuram-se como estratégias privilegiadas (López Pena, 2016a e 2016b).

Em relação com a comunidade local, há numerosos trabalhos a conceitualizar e estudar os impactes nela das políticas culturais e, sobretudo, das políticas turísticas, entendidas aqui como «um conjunto de atitudes e estratégias que um grupo organizado adota face ao turismo (ex. promoção, restrição ou extinção), derivando das políticas gerais e traduzindo-se em normas e regulamentos» (Pereiro \& Fernandes, 2018: 387). Nas primeiras, cobra força na atualidade entender a população local como mais um agente envolvido nas políticas culturais (Martinell \& López, 2007), para além dos tradicionais sector público, terceiro sector e organizações privadas. No âmbito do turismo, com mais percorrido e produção, habitualmente os estudos centram-se no «lado da procura» e menos no «lado da oferta» (Costa, 2005: 283).

Uma vez consolidados, com o apoio decisivo da OMT em 1999 (Pereiro \& Fernandes 2018), os discursos à volta do turismo como fenómeno sustentável (Remoaldo \& Cadima Ribeiro, 2017 ou Gascón 2016, este último para uma visão crítica sobre as possibilidades de salvaguardar o ambiente e, à vez, garantir o crescimento económico da atividade), os estudos dos impactes nas comunidades locais têm experimentado um aumento considerável em vários espaços geográficos (Scalabrini, Remoaldo \& Lourenço, 2014), podendo ser eles de signo positivo ou negativo nas suas dimensões económicas, socioculturais, ambientais (Remoaldo \& Cadima Ribeiro, 2017; Pereiro, 2009) e até simbólicas (considerados estes impactes simbólicos de acordo com a noção de capital simbó-

4 Repare-se em que «durante las cuatro legislaturas del gobierno del Partido Popular [PPdeG, regionalistas de centro-direita], el presupuesto de CRTVG [Compañía de Radio Televisión de Galicia, a emitir desde 24 de julho de 1985], Turismo y otras actividades no puramente relacionadas con la cultura, como la promoción del Camino de Santiago, tenían un peso presupuestario muy importante, absorbiendo el $50 \%$ de los recursos destinados a la Consellería de Cultura[, Comunicación Social y Turismo]» (Lage, Losada \& Gómez, 2012: 125). 
lico de P. Bourdieu [2001] ou, mais especificamente, com o conceito de sustentabilidade identitária de Torres Feijó [2015] $)$. Assim, os residentes são entendidos como atores, amiúde denominados stakeholders, parte interessada ou interveniente a ser considerada na análise do fenómeno turístico.

Vários são os fatores que, segundo a literatura, podem condicionar a perceção da comunidade local relativamente ao turismo. Em síntese de Felix et al (2017: 40-41):

O apoio dos residentes ao desenvolvimento do turismo está fortemente ligado a percepção dos impactos sociais, culturais, econômicos e ambientais estimulados por este fenômeno, como também é afetado pelas relações de poder e confiança entre a comunidade, atores governamentais e iniciativa privada [...], a imagem, seja afetiva e/ ou cognitiva, e o apego dos residentes a localidade [...], entre outros. Ressalta-se que as duas últimas dimensões citadas, ou seja, apego e imagem, são recentes no campo de estudo dos fatores que influenciam o apoio do residente e têm contribuído para o melhor entendimento do tema.

Neste sentido, a Teoria do Intercâmbio Social (Blau, 1968) preconiza a existência de uma relação (negociação) entre a perceção e atitudes da população local e a natureza do seu envolvimento com a indústria turística e os turistas (Andereck et al., 2005), que pode estar condicionada pela dependência económica do turismo ou, até, pela distância relativa aos locais com maior pressão turística. Apesar de surgirem estudos a visibilizar os efeitos negativos da massificação turística de alguns espaços (Delgado, 2007; Milano, 2017; Pereiro \& Fernandes, 2018) ou mesmo a pôr em questão, a partir da academia, a ideia de turismo sustentável (o referido Gascón, 2016), as análises dos impactes do fenómeno turístico tendem, em linha com os postulados da OMT, a estudar a perceção das comunidades locais em função das lógicas da indústria; i.e., o objetivo, de uma maneira geral, passa por conhecer os impactes numa dada comunidade com o intuito de melhorar e aumentar os fluxos turísticos e não, por exemplo, pela abordagem dos impactes do turismo na qualidade de vida dos locais (Andereck \& Nyaupane, 2010), ou mesmo, invertendo o foco, por contribuir para o entendimento e o reforço da identidade comunitária e do bem-estar da população local. Nesta direção, cabe apontar a inexistência de estudos para o caso de Compostela a abordar a perceção da comunidade local e com foco nela.

\section{METODOLOGIA}

Neste trabalho, como já foi indicado, utilizam-se duas metodologias complementares: em primeiro lugar realiza-se um levantamento analítico da bibliografia específica

$5 \quad$ «Identity sustainability is what guarantees the continuity of a community and the consensus about the community's identity, understanding sustainability in a two fold way: as the action of the community to keep the same items, and as the action of the community to preserve its identity and its limits» (Torres Feijó, 2015: 148). 
sobre as políticas públicas para a cultura e o turismo implementadas em Santiago de Compostela, com foco nos Caminhos de Santiago. Num segundo momento, com recurso a um corpus construído a partir de inquéritos passados a habitantes e comerciantes de Santiago de Compostela, é analisada a perceção dos impactes destas políticas na comunidade local. Para conhecermos empiricamente a perceção da comunidade local em relação com estas políticas públicas foram utilizadas duas bases de dados construídas no seio do projeto da Rede Galabra em que este trabalho está inserido, uma primeira composta por 929 inquéritos passados em junho, agosto e setembro, e entre de outubro e dezembro de 2014, assim como, finalmente, em abril de 2015 às pessoas residentes em Santiago de Compostela e a sua comarca (BD Locais); e outra base de dados com informação tirada dos 410 inquéritos passados a comerciantes da cidade entre março e abril de 2015 (BD Comerciantes). Entre estas duas bases foram comparadas as variáveis de estudo. A ficha técnica dos dados utilizados pode ser consultada na Tabela I colada abaixo.

Tabela I. Ficha técnica das Bases de Dados utilizadas

\begin{tabular}{|c|l|l|}
\hline FICHA TÉCNICA & Banco de Dados Locais & Banco de Dados Comerciantes \\
\hline Tipo de inquérito realizado & \multicolumn{2}{|c|}{ Pessoal com questionário estruturado } \\
\hline Universo & $\begin{array}{l}\text { Residentes em Santiago de } \\
\text { Compostela (ou em municípios } \\
\text { limítrofes, (Teo e Ames) }\end{array}$ & $\begin{array}{l}\text { Comerciantes que trabalham em } \\
\text { Santiago de Compostela }\end{array}$ \\
\hline Âmbito geográfico & \multicolumn{2}{|c|}{ Santiago de Compostela } \\
\hline População & Infinita $(>100.000)$ & 4.096 (marco de mostragem) \\
\hline Tamanho da mostra & 929 inquéritos válidos & 410 inquéritos válidos \\
\hline Erro de mostragem & $\pm 3,2 \%$ & $95 \% ; \mathrm{p}=\mathrm{q}=0,5$ \\
\hline Nível de confiança & \multicolumn{2}{|c|}{ De conveniência } \\
\hline Procedimento de mostragem & & 2015 \\
\hline Datas & $2014-2015$ & \multicolumn{2}{|c|}{95} \\
\hline
\end{tabular}

Fonte: elaboração própria.

As amostras estão compostas maioritariamente por mulheres (54\% da amostra de locais e $56 \%$ da de comerciantes), indo a idade das pessoas inquiridas de 16 a 91 anos, com uma média de 45 anos na amostra de locais e de 42 na de comerciantes. Quanto à formação regrada, a maioria dos inquiridos tem estudos superiores, quer formação profissional de grau superior ( $8,2 \%$ os locais e $16,5 \%$ os comerciantes) quer estudos universitários médios (34,3\% os locais e 34,1 os comerciantes) e superiores - incluídos aqui mestrado e doutoramento - (13,8\% dos locais e 4,4\% dos comerciantes). Já quanto à atividade profissional, $14,3 \%$ dos locais e $27 \%$ dos comerciantes depende economicamente do turismo (total ou parcialmente). A amostra de comerciantes está constituída por estabelecimentos 
distribuídos por toda a cidade 6 : 25,2\% localizados na parte antiga, $24,7 \%$ no ensanche, $12,1 \%$ nos parques empresariais do norte da cidade e $21,4 \%$ nas entradas dos caminhos de Santiago (10,1\% do português, 7,3\% do francês e 4,5\% do de Fisterra); o resto da cidade, incluído o rural (1,5\%), acolhe os demais locais comerciantes. O perfil das amostras pode ser consultado na Tabela II colada abaixo:

Tabela II. Perfis da população analisada

\begin{tabular}{|c|c|c|c|}
\hline \multicolumn{2}{|l|}{ PERFIS } & Locais & Comerciantes \\
\hline \multirow{2}{*}{ Género } & Mulheres & $54 \%$ & $56 \%$ \\
\hline & Homens & $46 \%$ & $44 \%$ \\
\hline \multicolumn{2}{|l|}{ Idade } & $\begin{array}{l}\text { Média } 46 \text { anos } \\
\text { (desvio padrão: } 17,7 \text { ) }\end{array}$ & $\begin{array}{l}\text { Média } 42 \text { anos } \\
\text { (desvio padrão: } 11,4 \text { ) }\end{array}$ \\
\hline \multirow{3}{*}{ Nível de Estudos } & Universitários Superiores & $13,8 \%$ & $4,4 \%$ \\
\hline & Universitários Médios & $34,3 \%$ & $34,1 \%$ \\
\hline & FP de grau superior & $8,2 \%$ & $16,5 \%$ \\
\hline Atividade Profissional & Dependência do turismo & $14,3 \%$ & $27 \%$ \\
\hline \multirow{5}{*}{$\begin{array}{l}\text { Distribuição } \\
\text { geográfica da } \\
\text { atividade Comercial }\end{array}$} & Parte Antiga & - & $25,2 \%$ \\
\hline & Ensanche & - & $24,7 \%$ \\
\hline & Parques Empresariais & - & $12,1 \%$ \\
\hline & Caminho de Santiago & - & $21,4 \%$ \\
\hline & Resto da cidade & - & $16,6 \%$ \\
\hline
\end{tabular}

Fonte: elaboração própria.

Dos inquéritos que estão na origem da informação recolhida nas duas bases de dados acima descritas foram selecionadas, para analisarmos a perceção da comunidade local em relação com os assuntos em foco, cinco perguntas presentes em ambos questionários (veja-se abaixo a Tabela III).

6 A cidade de Santiago está constituída por: a Parte Antiga; a Zona Nova - que nasceu nos anos 50 do século XX e cresceu nas décadas dos 60 e 70 com a construção do denominado "Ensanche"; os bairros periféricos; os parques industriais e comerciais; e as zonas tradicionais - algumas originalmente núcleos independentes e que foram sendo absorbidas pela dinâmica urbana. Em função dos objetivos definidos e a intensidade de trânsito de peregrinos, considerámos separadamente as entradas do Caminho Francês e Português, coincidentes com as entradas tradicionais à cidade pelo leste e pelo sul, respetivamente; e a saída ao Caminho de Fisterra - pelo oeste - a cada passo mais frequentado. 
Tabela III. Perguntas colocadas a Locais e Comerciantes de Compostela

\begin{tabular}{|l|l|}
\hline \multicolumn{2}{|c|}{ Perguntas Selecionadas (presentes na BD Locais e na BD Comerciantes) } \\
\hline Pergunta & Tipologia \\
\hline $\begin{array}{l}\text { Valore o seu grau de acordo com a imagem institucional de Santiago } \\
\text { de Compostela }\end{array}$ & \multirow{2}{*}{ Escala Likert de 8 pontos } \\
\cline { 1 - 2 } $\begin{array}{l}\text { Em que medida considera que o Caminho de Santiago representa } \\
\text { Santiago de Compostela }\end{array}$ & \\
\cline { 1 - 2 } $\begin{array}{l}\text { Em que medida considera que o Caminho de Santiago representa a } \\
\text { Galiza }\end{array}$ & \multirow{2}{*}{ Nominal dicotómica: sim/não } \\
\cline { 1 - 2 } Considera que em Compostela existem elementos importantes que \\
\cline { 1 - 2 } $\begin{array}{l}\text { Considera que uma parte dos espaços de Santiago deveriam ser } \\
\text { reservados ao turismo }\end{array}$
\end{tabular}

Fonte: elaboração própria.

Das cinco perguntas, coincidentes em ambos inquéritos, três correspondem-se com escalas tipo Likert de 8 pontos e duas com resposta nominal e dicotómica (sim/não). As respostas a estas perguntas foram processadas com o programa estatístico IBM SPSS vs. 24. Quanto à tipologia técnica das nossas análises, foram realizados estatísticos descritivos univariados para as variáveis de estudo. Em função do impacto dos interesses económicos dos indivíduos vinculados ao fenómeno turístico, foram realizados testes t de diferença das médias das escalas entre os comerciantes e os não comerciantes. As mesmas análises foram realizadas para comparar as médias das escalas entre os informantes que declararam depender do turismo e os que afirmaram não ter qualquer dependência económica. As diferenças intergrupo das variáveis nominais foram analisadas por meio de tabelas de contingência, e a sua significação foi abordada mediante testes Chi2 de Pearson.

\section{APRESENTAÇÃO DE RESULTADOS}

Apresentamos em primeiro lugar os resultados tirados das abordagens feitas sobre um volumoso corpus bibliográfico relativo às políticas culturais e turísticas referidas à cidade galega de Santiago de Compostela. Num segundo momento mostraremos os resultados tirados da informação empírica analisada sobre o grau de (des)acordo da comunidade local quanto a: 1) imagem institucional de Santiago de Compostela (pergunta 1); 2) representatividade do Caminho em relação com Santiago (pergunta 2) e a Galiza (pergunta 3); 3) perceção das políticas de comunicação institucional da cidade no exterior; e 4) aceitação/ impacto do turismo (pergunta 5). 


\subsection{As políticas culturais e turísticas em Santiago de Compostela}

Enquanto meta dos Caminhos de Santiago, a cidade de Santiago de Compostela alcançou, a partir das últimas décadas do século XX, uma vigorosa visibilidade a nível internacional inédita na época contemporânea (Simone Novello et al., 2013). De alguma forma, recuperou uma antiga capacidade de atração de origem medieval que, ultrapassando diferentes fases, tinha acabado por decair na época contemporânea (Lois \& López, 2012); à par, a cidade experimentou um forte reposicionamento nos mercados turísticos internacionais. Apesar de sustermos a centralidade do período que vai de 1980 até inícios de 2000 para entender, do ponto de vista das políticas culturais e turísticas, a nova posição da cidade, consideramos inelidível quando menos termos presentes dois momentos prévios que condicionam as ações do período citado: (i) a segunda inventio, em fins do século XIX e (ii) a sublimação nacional-católica durante a ditadura franquista.

A segunda inventio ${ }^{7}$, após o período de forte instabilidade surgido da irrupção do liberalismo, está caraterizada pela intervenção de um número restrito de agentes vinculados à Igreja católica, nomeadamente os arcebispos e cardeais compostelanos Payá y Rico (1875-1886) e Martín de Herrera (1888-1922). Na interpretação de Ramón Villares (2017) esta nova invenção surge determinada pelo apoio do Papa de Roma e o recurso à incipiente ciência forense, dando como resultado a autenticação em 1884 pelo Papa Leão XIII (com a bula Deus Omnipotens) de uns restos humanos encontrados na Catedral de Santiago como sendo os do Apóstolo Santiago o Menor. Por outro lado, em função do interesse da Igreja católica por revitalizar as peregrinações (Lourdes a partir de 1858, Montserrat, Covadonga, Fátima na década de 10 do século XX, etc.) são promovidas peregrinações sistemáticas a nível local e regional a Santiago (Santos Solla, 2006).

Após este primeiro impulso protagonizado quase exclusivamente pela Igreja católica, durante a ditadura franquista (de 1936 a 1975 na Galiza), inicia-se uma estreita colaboração entre a Igreja católica e o Estado (Santos \& Trillo-Santamaría, 2017). Entendemos haver um certo consenso à hora de identificar o interesse do regime, impulsando o caminho na sua dimensão religiosa, para legitimar as linhas de força do seu próprio ideário (Santos, 2006; Somoza \& González, 2017; Villares, 2017). Como se pode apreciar na Figura 1, este longo período está pontuado por diversas ações que, promovidas quase

$7 \quad$ A segunda inventio é continuadora terminologicamente do que se denominou, para o surgimento do fenómeno jacobeu, inventio:

el camino de Santiago tiene sus orígenes en el siglo IX cuando se descubre y se reconoce por parte de la monarquía y de la iglesia lo que se consideraron los restos del cuerpo del apóstol Santiago el Mayor. La difusión de esa noticia dio lugar en los siglos siguientes a un intenso proceso peregrinatorio desde todos los rincones de Europa. La vasta literatura sobre el tema ha desarrollado numerosos argumentos para justificar la trascendencia que llegó a tener este fenómeno. Éstos van desde las luchas de poder dentro del cristianismo hasta los más conocidos relacionados con la frontera frente al expansionismo del Islam. En cualquier caso, lo importante es que surge una ruta que va mucho más allá de las motivaciones simplemente religiosas y que, en cierta medida, recupera el espíritu europeista de Carlomagno figura a la que reiteradamente se ha querido vincular con el fenómeno jacobeo (Santos Solla, 2006: 136). 
exclusivamente pelo Estado, dotam o Caminho de Santiago e a própria cidade de Santiago de Compostela de diversas valências, seja simbólicas, seja de equipamentos, com o intuito de relançar a rota jacobeia dentro das coordenadas ideológicas do franquismo.

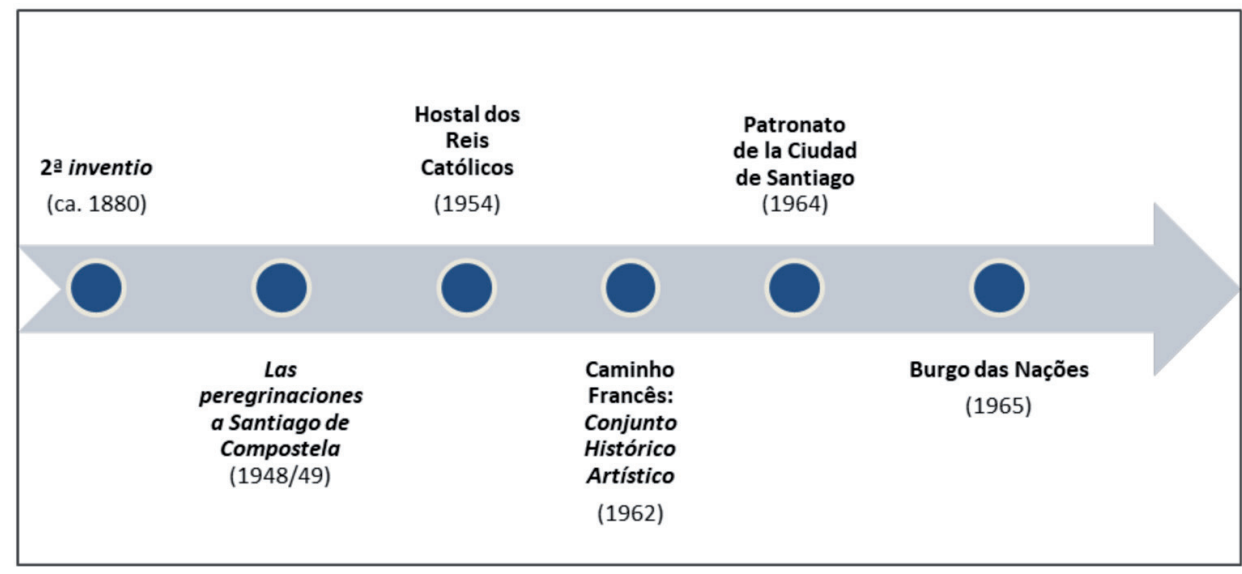

Figura 1. Ações de relançamento do Caminho de Santiago anteriores à reinventio.

Fonte: Elaboração própria.

Já a partir da década de 80 e até inícios do século XXI, têm lugar uma série ações turístico-culturais, na maioria dos casos, promovidas por um conjunto de atores poliédrico, com foco em Santiago de Compostela como meta dos Caminhos de Santiago, que enformam o que denominamos de reinventio (ou, seguindo a lógica terminológica em uso, terceira inventio; vid. Figura 2).

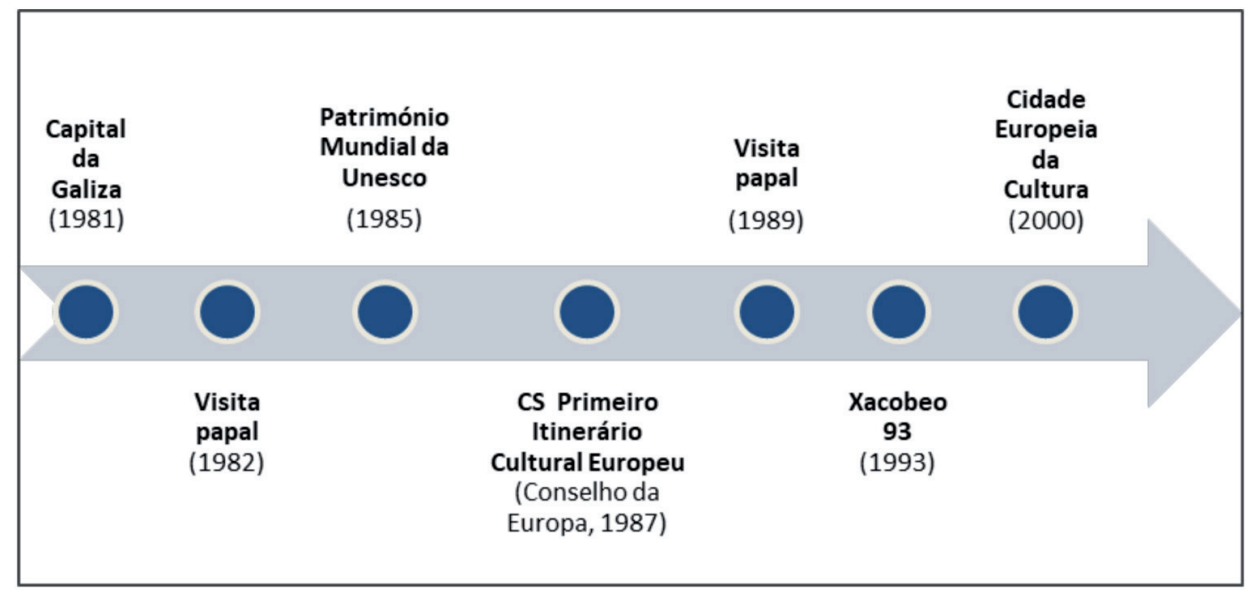

Figura 2. Eventos (político-)culturais da reiventio.

Fonte: elaboração própria. 
Sem nos determos aqui no relevantíssimo papel de alguma produção cultural elaboradora de narrativas centrais para entender os discursos - e as práticas - à volta da cidade e dos Caminhos - que aliás têm condicionado o fenómeno em estudo de múltiplas formas -, tais como os produtos de Paulo Coelho (particularmente o romance $O$ diário de um mago de 1987) (Torres Feijó, 2012; Rodríguez Prado, 2015; Fernández Rodríguez, del Río \& Rodríguez Prado, 2016; Fernández Rodríguez, 2016; Fernández Rodríguez \& Samartim, 2016; BelloVázquez, 2016; Somoza Medina \& Lois González, 2017), vários fatores conjunturais vão condicionar os discursos, as ideias e, em definitivo, a natureza da reinventio. Um destes fatores está vinculado à redinamização da construção europeia (da União Europeia) na década de 80 e ao interesse (de longo percurso) do Estado Espanhol por aderir ao projeto europeu (o qual se verifica com a adesão à Comunidade Económica Europeia, juntamente com Portugal, em 12 de junho de 1985). Nesta direção, são vários os discursos que alicerçam a ideia de Caminho e cidade como expressão de uma identidade europeia, útil aos interesses do governo espanhol (Santos \& Trillo-Santamaría, 2017), na altura objeto de intenso debate relativamente à dimensão religiosa. Nesse mesmo ano, a parte antiga de Santiago de Compostela era declarada Património Mundial da UNESCO (1985) e, em 1987, primeiro Itinerário Cultural Europeu pelo Conselho da Europa.

Paralelamente, desde os seus inícios, a reinventio esteve marcada também pelas tomadas de posição da Igreja católica. De facto, de acordo com Torres Feijó (2014: 293):

os diversos discursos enunciados [sobre a cidade e os Caminhos] correspondem a diversas estratégias, que podem ser sintetizadas, no caso das organizações [...] envolvidas, no apoio a um impulso proselitista da Igreja Católica, sobretudo dirigido às pessoas jovens e no alicerçar esta Igreja como base e fundamento da Europa e na vontade de articulação e consolidação europeias pela EU; e, igualmente, na proteção e valoração do património material e imaterial que a UNESCO persegue.

Antes da celebração do primeiro Congreso Internacional de Asociaciones Jacobeas (na cidade de Jaca, 1987), a Igreja católica tinha estabelecido, em 1971, 100 km. como distância mínima para ter direito à Compostela e, portanto, ser considerado peregrino aos seus olhos (Somoza \& Lois, 2017; Pereiro, 2018). Ainda sobre o papel da Igreja católica, destaca a intervenção explícita do papa João Paulo II, expressa em duas visitas à cidade (1982 e 1989, ambas na fase final da chamada Guerra Fria [1945-1991]). Segundo Elias Torres (2011: 396), a primeira destas visitas constitui a encenação do «novo discurso» da Igreja católica sobre o Caminho de Santiago e a própria cidade, alicerçado em: (i) a «caracterização de Santiago e, sobretudo, o Caminho e a peregrinação como um histórico elemento vertebrador da Europa»; (ii) «a identificação do cristianismo como raiz da identidade e a coesão europeias»; (iii) «a identificação de Santiago, do Apóstolo e do Caminho como meta e processo de conversão e superação católicas». Esta primeira visita à cidade ficaria também assinalada por um «acto europeista» (Santos, 2006: 138), no interior da catedral de Santiago e com participação do rei da Espanha na altura, Juan Carlos I de Bourbon. Interessa notar como, a partir deste momento, a Igreja católica (e 
os seus discursos e ideias) constitui-se como um agente principal das políticas culturais e turísticas na cidade enquanto meta dos Caminhos de Santiago.

Outro dos fatores determinantes que vão condicionar as políticas culturais e turísticas da cidade prendem-se com a construção do denominado Estado das Autonomias após aprovação da Constituição Espanhola de 1978. Neste quadro, a Comunidade Autónoma de Galicia, com capital oficial em Santiago de Compostela, inicia o seu percurso em 1981, também a implementar as suas próprias políticas culturais (Bouzada, 2008). A nova administração autonómica, principal ator cultural, investiu na década de 80 na «consolidación de las políticas culturales [...] para su uso simbólico como elemento constructor de la legitimidad política y social de la autonomia» (Lage, Losada \& Gómez, 2012: 122), convertendo-se, na prática, no agente dominante da vida cultural na Galiza (Lorenzo, 2017).

Por seu turno, o poder autárquico promoveu um ambicioso plano de desenvolvimento com centralidade cultural, em estreita colaboração com o governo autonómico. De facto, Santiago de Compostela constitui-se no âmbito autonómico como uma capital de nova criação, cujo novo estatus é discutido pela administração local da cidade da Corunha e, portanto, precisada de legitimidade, toda a vez que tinha sido «tradicionalmente postergada por la Administración del Estado a no ser cabeza de provincia. Compostela carecía de tradición de liderazgo nacional o infraestructura administrativa» (Lage, Losada \& Gómez, 2012: 116). A incipiente ansiedade de status de Santiago foi enfrentada, segundo Rodríguez González (2015: 379), com a «confluencia de un ayuntamiento empeñado en jugar la baza del patrimonio urbano y arquitectónico, y una administración autonómica que ideó [em 1993] un ambicioso programa de promoción cultural y económica (a partir del turismo) en torno al discurso del Camino de Santiago: el Xacobeo».

O Xacobeo explica em grande medida os picos de afluência de peregrinos em cada Ano Santo desde a sua primeira edição em 1993 (vid Figura 3), configurando-se como o elemento catalisador dos diversos atores e discursos que sobre Santiago de Compostela e os Caminhos começam a operar até à atualidade. O Xacobeu, promovido pela administração autonómica presidida por Manuel Fraga Iribarne (PPdeG, 1989-2005)², contou com um forte investimento público, até o ponto de as despesas em cultura alcançarem entre 1986 e 2005 um crescimento de $1.200 \%$. A partir desse momento os orçamentos dedicados à cultura pela administração autonómica

van depender extraordinariamente da proximidade ou distancia respecto de cada un dos Xacobeos [...] Esa celebración serviu de pretexto para que se lle prestasse unha

8 "El Plan Xacobeo fue aprobado por unanimidad en el Parlamento de Galicia en 1991 con la misión de armonizar los esfuerzos de las fuerzas políticas, civiles y eclesiásticas para conseguir la recuperación física, cultural y económica del Camino de Santiago. Y todo ello con un triple objetivo: 1) Reafirmar la identidad de Galicia a través de un elemento esencial de su historia y personalidad. 2) Vincular Galicia y el Camino de Santiago con la idea de Europa. 3) Promocionar a Galicia de forma global en cada Año Santo Compostelano" (http://www.fundacioncontemporanea.com/pdf/Publica11._Xacobeo2010._ Ignacio_Santos.pdf). 
atención moi especial á promoción do Camiño de Santiago e ao Xacobeo como evento galego de referencia. Unha estratexia que foi despregada co fin de mellorar a proxección exterior e a imaxe de Galicia para poñela ao servizo do desenvolvimento turístico do país» (Bouzada, 2008: 46-47).

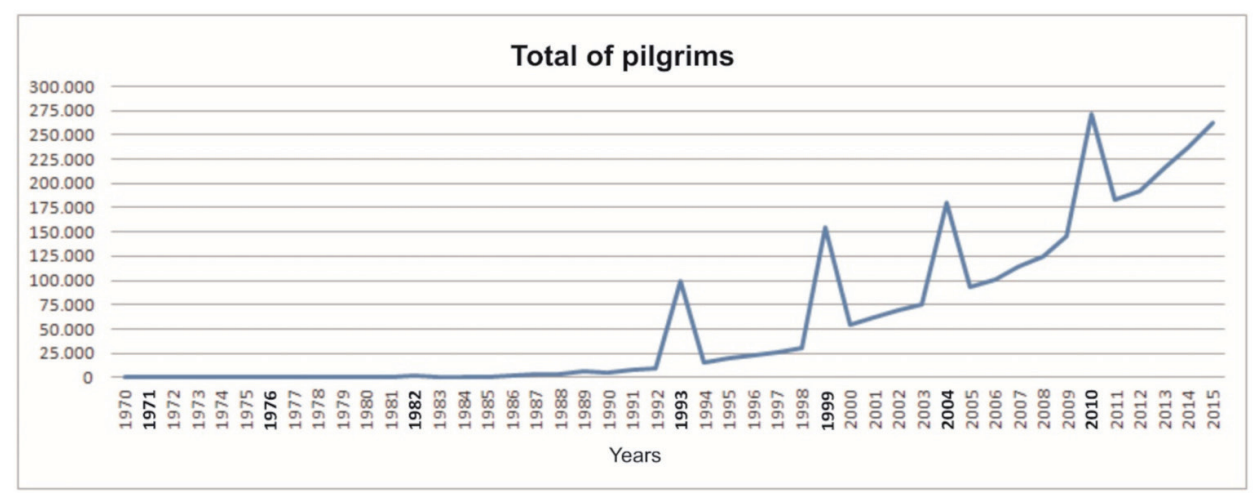

Figura 3. Afluência de pessoas peregrinas a Compostela.

Fonte: Somoza Medina \& Lois González, 2017: 50.

O Xacobeo acaba por funcionar, então, «como vehículo y expresión máxima del desarrollo turístico de Galicia» (Santos Solla, 1999: 116) e nele participam, direta ou indiretamente, uma série de atores cujos discursos se caraterizam por uma relativa unanimidade quanto a ideias e propósitos. À volta dele vai surgindo um conjunto de organizações, tais como a Sociedade Anónima para a Xestión do Xacobeo (1991) ou o Consorcio da Cidade de Santiago (1992), «órgano executor do Real Padroado [da Cidade] de Santiago [de Compostela -criado em 1991 e integrado por membros do Estado, o Governo galego, o concelho, a universidade e o arcebispado-], no que se materializa a cooperación institucional entre o Goberno de España, a Xunta de Galicia e o Concello de Santiago» (http:// www.consorcio-santiago.org/). O Consórcio contribui decisivamente para dotar a cidade de uma série de equipamentos turístico-culturais como o Palácio de Congressos, o Multiusos do Sar, o Pavilhão de Galiza na Exposição Universal de Sevilha 92 ou o Complexo do Monte do Gozo (Celeiro, 2013).

Outro dos atores citados, a Universidade de Santiago de Compostela (USC), assinte nesse ano 1993 à constituição do Grupo Compostela e, mais recentemente, em 2016, cria uma Cátedra do Caminho e das Peregrinações apoiada financeiramente pelo governo autónomo galego e fruto de um protocolo entre a instituição universitária, a Axencia de Turismo de Galicia (Turgalicia, criada em 2012) e a Catedral de Santiago.

Aliás, é a partir da primeira celebração do Xacobeo em 1993 quando se precipitam os reconhecimentos institucionais de vário tipo à cidade e ao Caminho: declaração do Caminho de Santiago Património da Humanidade (1993), prémio Europa Nostra (1994, 1996 e 2001), Capitalidade Europeia da Cultura em 2000 (eleita em 1995), Medalha da 
Assembleia Parlamentar do Conselho da Europa (1997) ou o Premio Príncipe de Asturias a la Concordia (2004).

Do ponto de vista turístico e de capacidade de atração de visitantes (peregrinos explicitamente; vid atrás a Figura 3), o caso de Santiago de Compostela é valorizado pela administração galega como uma história de sucesso, tal como indica o atual presidente autonómico, Alberto Núñez Feijóo (2013: 4):

Hai un dobre éxito nesa tarefa que conxuntamente emprenden na década dos 90 a Igrexa Católica e a Xunta de Galicia, presidida entón por Don Manuel Fraga e sendo conselleiro de Cultura Víctor Manuel Vázquez Portomeñe. Conseguen primeiramente converter o Camiño en faro que aluma a relixiosidade e a espiritualidade europea, e despois renóvano para que ese faro non se apague nunca [...] Compostela é o corazón espiritual de Europa, o Camiño o seu arteria principal.

Ora, o certo é que a bibliografia analisada evidencia a existência de uma ampla margem para a discussão deste alegado êxito, nomeadamente quanto ao modelo de cidade em construção ( «un lugar pensado para turistas y para la industria de servicios asociada, pero poco atractivo para vivir», García Vázquez, 2009: 8); às práticas de visitantes e, nomeadamente, de locais; ao real impacto económico; o apagamento de outras dimensões citadinas; etc. (Santos Solla, 1999 e 2006; Monterroso, 2011; Torres Feijó, 2014; Bello, 2015; Carral, Carreira, Vila \& Torres Feijó, 2016; Somoza \& Lois, 2017; Martin-Duque, 2017).

\subsection{A perceção da comunidade local}

As perceções do «lado da procura» foram derivadas das respostas proporcionadas pelas duas mostras de inquiridos no relativo aos assuntos em foco - cinco perguntas presentes em ambos os questionários. Os estatísticos descritivos univariados do conjunto das perguntas formuladas são apresentados na Tabela IV e são comentados separadamente a seguir.

Tabela IV. Estatísticos descritivos univariados do conjunto da mostragem

\begin{tabular}{|l|c|c|c|c|}
\hline \multirow{2}{*}{ Pergunta } & \multicolumn{2}{|c|}{ Locais } & \multicolumn{2}{c|}{ Comerciantes } \\
\cline { 2 - 5 } & Média & Desvio Padrão & Média & Desvio Padrão \\
\hline $\begin{array}{l}\text { Valore o seu grau de acordo com a imagem } \\
\text { institucional de Santiago de Compostela }\end{array}$ & 5,66 & 1,906 & 4,70 & 2,335 \\
\hline $\begin{array}{l}\text { Em que medida considera que o Caminho de } \\
\text { Santiago representa Santiago de Compostela }\end{array}$ & 6,81 & 1,748 & 7,22 & 1,484 \\
\hline $\begin{array}{l}\text { Em que medida considera que o Caminho de } \\
\text { Santiago representa a Galiza }\end{array}$ & 7,26 & 1,479 & 7,56 & 1,156 \\
\hline $\begin{array}{l}\text { Considera que em Compostela existem elemen- } \\
\text { tos importantes que não se conhecem fora. }\end{array}$ & \multicolumn{3}{|c|}{ Frequência de sim } \\
\cline { 2 - 5 } & \multicolumn{4}{|c|}{$53 \%$} \\
\hline $\begin{array}{l}\text { Considera que uma parte dos espaços de Santia- } \\
\text { go deveriam ser reservados ao turismo. }\end{array}$ & \multicolumn{3}{|c|}{$6,8 \%$ cia de sim } \\
\cline { 2 - 5 }
\end{tabular}

Fonte: elaboração própria. 
a) Imagem institucional de Santiago de Compostela («Valore o seu grau de acordo com a imagem institucional de Santiago de Compostela»):

Yolanda García Vázquez (2009: 7) afirmava em 2009 que

de la observación detenida de los documentos existentes para difundir la imagen externa de la ciudad, la bibliografía y folletos turísticos, se constata reiteradamente un énfasis en la monumentalidad del casco histórico de Santiago, sus infraestructuras culturales, educativas, etc., y en los premios de excelencia internacional recibidos por su patrimonio y política urbanística. La imagen fomentada es la de ciudad cultural, cosmopolita, abierta, universitaria, moderna y contemporánea, donde conviven la tradición y la innovación.

Para esta investigadora existia entre a população local, aliás, um alto grau de conformidade com esta imagem projetada desde as instituições: «los ciudadanos residentes y medios de comunicación aceptan y participan de un discurso mediático poderoso que beneficia el status quo político» (Garcia Vázquez, 2009: 8; itálicos no original).

Por seu lado, os nossos dados indicam que o grau de acordo com a imagem institucional de Santiago não atinge o "aprovado", a levarmos em conta que na escala utilizada o ponto médio está entre 5 e 6 , e que na média das respostas a esta questão $(5,35)$ não chega à metade da escala. Esta média, se atendermos ao valor do desvio padrão, não representa a opinião do conjunto das pessoas consultadas.

\section{b) Perceção das políticas de comunicação institucional da cidade no exterior («Con-} sidera que em Compostela existem elementos importantes que não se conhecem fora»):

As respostas a esta pergunta são coerentes com o resultado anterior: uma ampla maioria dos inquiridos julga alguns dos elementos importantes de Santiago como não conhecidos fora. A falta de consenso quanto ao acordo com as políticas de comunicação institucional pode ser devido a que a imagem transmitida seja percebida como incompleta.

c) Representatividade do Caminho em relação com Santiago e com a Galiza («Em que medida considera que o Caminho de Santiago representa Santiago de Compostela/ Galiza»):

A ideia de o Caminho de Santiago representar a cidade é a que atinge uma valorização média mais alta e um menor desvio padrão; quer dizer, maioritariamente as pessoas inquiridas concordam quase totalmente com esta proposta. Algo similar acontece com a pergunta sobre a medida em que o Caminho representa a Galiza. A média é levemente menor e o desvio um bocado mais alto que para a questão anterior, mas os dados permitem fazer a mesma leitura: os inquiridos concordam maioritariamente em valorizarem muito positivamente esta proposta. Em conjunto, as respostas revelam uma perceção do Caminho enquanto elemento essencial da configuração da imagem própria entre os locais. 
d) Aceitação/ impacto do turismo («Considera que uma parte dos espaços de Santiago deveriam ser reservados ao turismo»):

A importância relativa do turismo para a cidade tal e como os locais a percebem foi medida de maneira extrema, propondo a possibilidade de reservar espaços exclusivos para o uso dos turistas: mais do $90 \%$ dos questionados rejeita esta sugestão e não concorda com delimitar os espaços da cidade em função da natureza dos indivíduos (local vs. visitante).

Já numa análise conjunta das três questões mais produtivas para os grupos de estudo (acordo com a imagem institucional e representatividade do caminho para Compostela e para a Galiza), verificamos que os testes t de diferença de médias não mostram diferenças significativas entre as médias das escalas nos grupos dependentes e não dependentes do turismo. A unanimidade é manifesta entre grupos. Face a isto, da divisão da mostra entre locais comerciantes e não comerciantes sim resultam médias significativamente $(\mathrm{p}<0,001)$ diferentes para as três escalas (Tabela V).

Tabela V. Significação da prova t de diferença de médias

\begin{tabular}{|c|c|c|c|c|c|c|}
\hline & & $\mathrm{F}$ & Sig. & $t$ & gl & Sig. \\
\hline \multirow{2}{*}{$\begin{array}{l}\text { Acordo com } \\
\text { a imagem } \\
\text { institucional }\end{array}$} & $\begin{array}{l}\text { Assumem-se } \\
\text { variações iguais }\end{array}$ & 35,465 & ,000 & 7,626 & 1239 & ,000 \\
\hline & $\begin{array}{l}\text { Não se assumem } \\
\text { variações iguais }\end{array}$ & & & 7,060 & 616,326 & , 000 \\
\hline \multirow{2}{*}{$\begin{array}{l}\text { O Caminho faz } \\
\text { parte da identidade } \\
\text { galega }\end{array}$} & $\begin{array}{l}\text { Assumem-se } \\
\text { variações iguais }\end{array}$ & 17,606 &, 000 & $-4,164$ & 1299 &, 000 \\
\hline & $\begin{array}{l}\text { Não se assumem } \\
\text { variações iguais }\end{array}$ & & & $-4,429$ & 901,417 &, 000 \\
\hline \multirow{2}{*}{$\begin{array}{l}\text { O Caminho faz } \\
\text { parte da identidade } \\
\text { de Santiago }\end{array}$} & $\begin{array}{l}\text { Assumem-se } \\
\text { variações iguais }\end{array}$ & 27,109 & ,000 & $-3,592$ & 1291 & , 000 \\
\hline & $\begin{array}{l}\text { Não se assumem } \\
\text { variações iguais }\end{array}$ & & & $-3,935$ & 976,062 &, 000 \\
\hline
\end{tabular}

Fonte: elaboração própria.

Como se observa na Tabela VI, o acordo com a imagem institucional é médio-alto entre os locais que não se dedicam profissionalmente ao comércio (5,66 sobre 8 pontos). Os comerciantes demostram um grão de acordo menor (4,70 sobre 8 pontos). Observa-se, portanto, uma certa quebra da unanimidade entre estes grupos de população, com um desacordo maior no grupo dos comerciantes. Por outro lado, a ideia de o Caminho fazer parte da identidade de Compostela e da Galiza é unanimemente aceite, apesar de no grupo de comerciantes a identificação ser maior (7,22 face a 6,80 - a primeira - e 7,56 face a 7,26 -a segunda). 
Tabela VI. Médias das escalas com diferenças significativas entre grupos

\begin{tabular}{|l|l|c|}
\hline \multirow{2}{*}{ Acordo com a imagem institucional } & Não comerciantes & 5,66 \\
\cline { 2 - 3 } & Comerciantes & 4,70 \\
\hline \multirow{2}{*}{ O Caminho faz parte da identidade galega } & Não comerciantes & 6,81 \\
\cline { 2 - 3 } & Comerciantes & 7,22 \\
\hline \multirow{2}{*}{$\begin{array}{l}\text { O Caminho faz parte da identidade de } \\
\text { Santiago }\end{array}$} & Não comerciantes & 7,26 \\
\cline { 2 - 3 } & Comerciantes & 7,56 \\
\hline
\end{tabular}

Fonte: Elaboração própria.

Quando a mostra foi dividida entre residentes dependentes e não dependentes do turismo não se obtiveram resultados significativamente diferentes entre grupos em nenhuma das variáveis $(\mathrm{p}<0,05)$. A divisão da mostra segundo a dedicação profissional ao comércio dos questionados sim mostrou resultados significativos $(\mathrm{p}<0,01)$ para a deteção de elementos desconhecidos para os visitantes (Tabela VII).

Tabela VI. Significação da Chi2

\begin{tabular}{|l|l|l|}
\hline & Valor & $\begin{array}{l}\text { Significação assintótica } \\
\text { (bilateral) }\end{array}$ \\
\hline Chi-quadrado de Pearson & $\begin{array}{l}16 \\
, 050 \mathrm{a}\end{array}$ &, 000 \\
\hline
\end{tabular}

Fonte: Elaboração própria.

Na tabela de contingência (Tabela VIII) percebe-se como a existência de elementos próprios de Santiago que não conhecem os visitantes está relativamente generalizada entre os locais não comerciantes (o 65,5\% deles assim o considera). Entre os comerciantes pouco mais da metade os deteta $(53 \%)$.

Tabela 8. Tabela de contingência elementos desconhecidos * comerciantes

\begin{tabular}{|c|c|c|c|c|}
\hline & & \multicolumn{2}{|c|}{ Elementos desconhecidos } & \multirow{2}{*}{ Total } \\
\hline & & Sim & Não & \\
\hline \multirow{2}{*}{ Não comerciante } & Reconto & 531 & 280 & 811 \\
\hline & $\%$ dentro de não comerciante & $65,5 \%$ & $34,5 \%$ & $100,0 \%$ \\
\hline \multirow{2}{*}{ Comerciante } & Reconto & 185 & 164 & 349 \\
\hline & $\%$ dentro de comerciante & $53,0 \%$ & $47,0 \%$ & $100,0 \%$ \\
\hline
\end{tabular}

Fonte: elaboração própria. 


\section{DISCUSSÃO DE RESULTADOS E SÍNTESE CONCLUSIVA}

As políticas culturais e turísticas, no período que vai de 1980 até o momento atual, são entendidas aqui, sob a denominação reinventio, como um processo relativamente rápido de ressignificação de Santiago de Compostela enquanto, não apenas mas fundamentalmente, meta dos Caminhos de Santiago. Com o concurso decisivo da Igreja católica e outras organizações internacionais, a reinventio foi promovida por agentes institucionais - Estado, Xunta da Galiza e Concelho - interessados em impulsar uma série de ideias relativas a: (i) a condição europeia dos Caminhos e de Santiago de Compostela/Galiza/ Espanha; (ii) a religião/espiritualidade como elemento estruturante dos territórios e das comunidades; (iii) o par património/cultura como um dos eixos centrais das políticas públicas; e (iv) o desenvolvimento do turismo como aspiração central.

Interessa notar particularmente como a reinventio é sustentada com solidez por uma evidente unanimidade; i.e., os vários atores envolvidos têm colaborado expressivamente com ancoragem em interesses diversos mas proficuamente articulados. Contudo, fora algum precedente surgido do independentismo galego não institucional (AMI, 2012), existem indicadores que demostram alguma erosão da fortaleza desta unanimidade já no âmbito político institucional, nomeadamente desde que uma nova opção partidária no âmbito da esquerda - Compostela Aberta (2015: 56) - alcança a Presidência da Câmara Municipal em 2015 e coloca no debate, por «exemplo: [a] regulación de prazas hoteleiras no casco histórico». Repare-se ainda, neste sentido, na proposta institucional de habilitar uma taxa para excursionistas, debatida no processo de elaboração do Plano de Xestión da Cidade Histórica de Santiago de Compostela (2018), ou, sobretudo, na falta de unanimidade manifesta relativa ao último grande projeto em matéria cultural da administração Fraga, a Cidade da Cultura (por exemplo Bouzada, 2008 ou Lage, Losada \& Gómez, 2012).

Por seu turno, os dados referidos à perceção da comunidade local mostram, em termos gerais, igualmente essa unanimidade. Assim, não se obtêm diferenças significativas entre os grupos de comerciantes/não comerciantes na variável reserva de espaços para o turismo; mais de $93 \%$ dos consultados considera que não há que reservar espaços. $\mathrm{O}$ dado, contudo, deverá ser contrastado com os reais usos dos espaços citadinos mais impactados pelo fenómeno turístico por parte da comunidade local, pois poderíamos estar a assistir a uma desistência inconsciente de, por exemplo, a parte antiga da cidade, outrora espaço privilegiado de sociabilidade local (Torres Feijó, 2014).

Sim resultam diferenças significativas entre locais comerciantes e não comerciantes nas outras quatro variáveis analisadas: (i) há acordo médio-alto com a imagem institucional entre não comerciantes e médio-baixo entre comerciantes - os comerciantes são mais críticos; (ii) a identificação do Caminho com Santiago é muito alta nos dois grupos, porém mais expressiva entre comerciantes; (iii) a identificação do Caminho com Galiza é também alta, mas menos se comparada com o ítem anterior e, no entanto, os comerciantes também associam mais o Caminho à Galiza. 
Em última instância, apenas o dado de $67,1 \%$ dos inquiridos considerarem haver elementos importantes da cidade como não conhecidos fora (com menos expressão entre os comerciantes) é que contraria a lógica da unanimidade da comunidade local face à reinventio. Neste ponto, cabe hipotetizar um desencontro com a imagem construída e promovida de Santiago de Compostela que, inevitavelmente, condiciona as ideias e práticas dos visitantes e, igualmente, dos locais.

Assim sendo, os dados indiciam, no seu conjunto e apesar dos matizes expostos, uma população local a assumir a unanimidade dos agentes identificados; e desinteressada, a priori, em problematizar e repensar a planificação cultural/turística da cidade. Poderia pensar-se, nesta linha de análise, na fortaleza dos discursos institucionais - e das suas estratégias e mecanismos de comunicação - acerca do modelo de cidade e do turismo, particularmente neste caso; por esta via, parece necessário explorar quais são os benefícios sentidos pelos santiagueses e, paralelamente, os lucros ou benefícios reais recebidos pelos mesmos. Os dados, nesta direção, apontam para uma clivagem reveladora quanto às perceções no par comerciante/não comerciante que, em sintonia com a literatura invocada, mostram dois grupos de locais com sensibilidades similares mas não exatamente coincidentes.

Em definitivo, parece possível afirmar que a reinventio, enquanto processo de ressemantização de Santiago de Compostela articulado em função de ambiciosas (e custosas) políticas culturais e, simultaneamente, turísticas, alicerçou-se no período em análise numa ampla unanimidade entre os agentes envolvidos e entre a população local, não sem excepções, neste caso, que caberá analisar mais em pormenor em próximos trabalhos. 


\section{BIBLIOGRAFIA}

AMI [Assembleia da Mocidade Independentista] (2012): A Terra nom se vende. O turismo é colonialismo. Dossier acessível em https://issuu.com/amigz/docs/livro-a_terra_nom_se_vende (26/06/2018).

Andereck, K.; Valentine, K.; Knopf, R.; \& Vogt, Ch. (2005): "Residents' perceptions of community tourism impacts". Annals of Tourism Research, Volume 32, Issue 4, October, Pages 1056-1076 https://www.sciencedirect.com/science/article/abs/pii/ S0160738305000770 (26/06/2018).

Andereck, K. \& Nyaupane, G. (2010): "Exploring the Nature of Tourism and Quality of Life Perceptions among Residents”, Journal of Travel Research, 50: 248-260.

Barbieri, Nicolás (2014): "Cultura, políticas públicas y bienes comunes: hacia unas políticas de lo cultural”, kult-ur. Revista interdisciplinària sobre la cultura de la ciutat, vol. 1, nº1, pp. 101-119, http://www.e-revistes.uji.es/index.php/kult-ur/article/ view/1255/0 (15/06/2018).

Bello Vázquez, Raquel (2015): A existência de uma comunidade luso-galega como elemento afetivo nas visitas a Santiago de Compostela procedentes de Portugal. Primeiros resultados através de inquéritos quantitativo-qualitativos. Em Torres Feijó, Bello Vázquez, Samartim \& Brito-Semedo (eds.), Estudos da AIL em Teoria e Metodologia Relacionamento nas Lusofonias II (pp. 87-96), Santiago de Compostela/ Coimbra, Associação Internacional de Lusitanistas.

Bello Vázquez, Raquel (2016): Consumos Literários, Práticas Culturais e Habitus: O Paradoxo de Paulo Coelho e o Caminho de Santiago, IS Working Papers, 3. a Série, N. 36 , http://isociologia.up.pt/sites/default/files/working-papers/wp36_160915100345. pdf $(26 / 06 / 2018)$.

Blau, P. M. (1968): “Interaction: social exchange”, in D. L. SILLS (ed.): International Encyclopaedia of the Social Sciences, Free Press, Glencoe, vol. 7, 1968, págs. 452457.

Bourdieu, Pierre (2001): O Poder Simbólico, 4ª ed., Viseu, Difel [1989].

Bouzada Fernández, Xan (2007): As políticas culturais nas sete principais cidades galegas. Consello da Cultura Galega http://consellodacultura.gal/mediateca/documento.php?id=111 (26/06/2018).

Bouzada Fernández, Xan (2008): "Políticas culturais e desenvolvemento económico: algunhas anotacións sobre a situación galega”, RIPS. Revista de Investigaciones Políticas y Sociológicas, Vol. 7, núm. 1, 31-53

Canelas Rubim, A. A. (2009): "Políticas culturais e novos desafios". Matrizes, v. 2, n. 2, 93-115, https://www.periodicos.usp.br/matrizes/article/view/38226 (28/06/2018).

Carral V., Emilio, Carreira, X. Carlos, Vila, Breogão M. \& Torres Feijó, Elias (2016): "A inter-relação entre gastronomia, cultura, turismo e comércio local em Santiago de Compostela. Primeiros resultados", in Tibério, L. M. et al. (orgs.), III Seminário "Alimentos e Manifestações Culturais Tradicionais"/II Simpósio Internacional 
“Alimentação e Cultura: Tradição e Inovação na Produção e Consumo de Alimentos" (pp. 263-280), Universidade de Trás-os-Montes e Alto Douro.

Celeiro Álvarez, Luis (2013): "Xacobeo 93. El renacer del Camino", in Simone Novello et al. (eds.). Xacobeo de un recurso a un evento turístico global, Santiago de Compostela: Andavira.

Compostela Aberta (2015): Gañemos un concello aberto. Programa. http://compostelaaberta.org/wp-content/uploads/2015/05/programaCompostelaAberta.pdf $(26 / 06 / 2018)$.

Costa, Carlos (2005): “Turismo e cultura: avaliação das teorias e práticas culturais do sector do turismo (1990-2000)", Análise Social, [online]. 2005, n.175, pp.279295. http://www.scielo.mec.pt/scielo.php?script=sci_arttext\&pid=S000325732005000300002\&lng=pt\&nrm=iso (28/06/2018).

Delgado, Manuel (2007): “Ciudades sin ciudad. La tematización 'cultural' de los centros urbanos", in David Lagunas (ed.), Antropología y turismo, Universidad Autónoma del Estado de Hidalgo, Pachuco de Soto, 2007, pp. 91-108.

EY (2015): Tempos de Cultura. O primeiro mapa global das indústrias culturais e criativas. Www.cisac.org/Media/Studies-and-Reports/Studies/EYCulturalTimes2015_ PT (28/06/2018).

Felix, J. P. S.; Chagas, M. M.; Marques, S. M.; \& Silva, V. H. (2017): “Análise da relação entre dependência econômica e percepção dos residentes sobre os impactos dos residentes sobre os impactos do desenvolvimento turístico da praia da Pipa/RN". Applied Tourism, 2(3), 37-64.

Fernández Rodríguez, M. L. (2016): Discursos sobre Santiago de Compostela y el/los Camino(s) de Santiago enla novela española actual (2010) a través de técnicas analíticas digitales: Posibilidades y valor Del conocimiento generado, Santiago de Compostela, Universidade de Santiago de Compostela (Tese de Doutoramento orientada por Elias J. Torres Feijó e Roberto Samartim).

Fernández Rodríguez, M. L., Río Araújo, M. del \& Rodríguez Pardo, F. (2016): “Dos productos y un destino: The Way (2010), Diario de un Mago (1987) y la imagen de Ga-

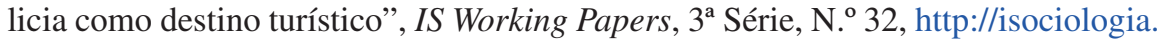
up.pt/sites/default/files/working-papers/wp32_160902115811.pdf (24/09/2017).

Fernández Rodríguez, M. L. \& Samartim, R. (2016): “Impacto dos discursos literários nas práticas e consumos culturais ligados aos Caminhos de Santiago: de Paulo Coelho aos souvenirs de cavaleiros templários", IS Working Papers, $3^{\text {a }}$ Série, N. ${ }^{\circ}$ 37 http://isociologia.up.pt/sites/default/files/working-papers/wp37_161014113435. pdf (24/09/2017).

García Vázquez, Y. (2009): "La ciudad como espectáculo. Marketing territorial, internet y atracción turística en Santiago de Compostela. ¿Éxito o fracaso?”, UOC Papers. Revista sobre la sociedad del conocimiento, 8, 1-10.

Gascón, J. (2016): “¿El turismo sostenible es un gremlin? De la autogestión local a la gentrificación”. Ecología política, № 52, 35-42. 
INVESTBRAGA (2014): Plano estratégico para o desenvolvimento económico de Braga 2014-2026 https://www.investbraga.com/files/strategicplans/planoestrategicoeconomicobraga_1.pdf (28/06/2018).

Lage, X., Losada, A. \& Gómez, M. (2012): “La política cultural en la comunidad autónoma gallega: de la dependencia a la autonomía", RIPS, Vol. 11, 3, 115-148.

Lois González, R.C. \& Lopez, L. (2012): "El Camino de Santiago: una aproximación a su carácter polisémico desde la geografía cultural y el turismo". Documents d'anàlisi geográfica, vol. 58, № 3, 459-479.

Lopes, João Teixeira (2010): "Da cultura como locomotiva da cidade-empresa a um conceito alternativo de democracia cultural", in Santos, M. de L. \& Pais, J. (orgs.), Novos Trilhos Culturais. Práticas e Políticas (pp. 51-61). Lisboa, Instituto de Ciências Sociais da Universidade de Lisboa.

López Palomeque, F. (2015): "Barcelona, de ciudad con turismo a ciudad turística. Notas sobre um proceso complejo e inacabado". Documents d'anàlisi geogràfica, Vol. 61, $\mathrm{N}^{\mathrm{o}} 3,483-506$.

Pereiro Pérez, X. (2016a): La verbena (en)cubierta: las actuaciones musicales en salas con programación periódica a través de la prensa local de Vigo (noviembre 1975-agosto 1990). Santiago de Compostela: Universidade de Santiago de Compostela. (Tese de doutoramento).

Pereiro Pérez, X. (2016b): Unha introdución á programación dos espectáculos de música popular en directo en Galicia. O exemplo dos macroconcertos en Vigo (2011). Santiago de Compostela: Universidade de Santiago de Compostela. (Trabalho de Fim de Mestrado).

Lorenzo, M. (2017): “As políticas culturais en Galicia”, Grial, n 215, 61-73

Martin-Duque, C. (2017): "Los impactos del turismo en el Camino de Santiago Francés: una aproximación cualitativa". Methaodos. revista de ciencias sociales, 5 (1), 62-73.

Martinell, A. \& López, T. (2007): Políticas culturales y gestión cultural: Organum sobre los conceptos clave de la práctica profesional. Girona, Documenta Universitaria.

Milano, C. (2017): Over tourism and Tourismphobia: Global trends and local contexts. Barcelona: Ostelea School of Tourism \& Hospitality.

Monterroso Montero, Juan M. (2011): "El Centro Histórico. La creación de una conciencia cultural. El caso de Santiago de Compostela" in Manuel Joaquim Moreira da Rocha (coord.). Actas do Seminário Centros Históricos: Passado e Presente. Porto, Universidade do Porto, pp. 39-69.

Núñez Feijóo, A. (2013): Prólogo, in Simone Novello et al (eds.). Xacobeo de un recurso a un evento turístico global, Santiago de Compostela, Andavira.

OMT (2014): El primer Congreso Internacional de la OMT sobre Turismo y Peregrinaciones explora el nexo entre el turismo y las rutas espirituales. Madrid, Organización Mundial del Turismo http://media.unwto.org/es/press-release/2014-09-16/ el-primer-congreso-internacional-de-la-omt-sobre-turismo-y-peregrinaciones(26/06/2018). 
OMT (2017): UNWTO Annual Report 2016, Madrid, UNWTO http://cf.cdn.unwto.org/ sites/all/files/pdf/annual_report_2016_web_0.pdf (24/09/2016).

OMT (2018): Tourism and Culture Synergies. https://www.e-unwto.org/doi/ pdf/10.18111/9789284418978 (26/06/2018).

Paül i Agustí, D. (2013): "Las políticas culturales y sus repercusiones en la imagen de la ciudad”. Scripta Nova, Vol. XVII, núm. 432, http://www.ub.edu/geocrit/sn/sn-432. $\operatorname{htm}(31 / 01 / 2018)$.

Pereira, Varico da Costa \& Peres, Américo Nunes (2010): Turismo Cultural e Religioso em Braga e Santiago de Compostela: Proposta de Criação de um Produto Conjunto", Revista Turismo \& Desenvolvimento, n.13/14, 677-686.

Pereiro Pérez, X. (2009): Turismo cultural: Uma visão antropológica. ed. 1, 1 vol., Tenerife, Pasos [E-book].

Pereiro Pérez, X. (2018): “Turismo y peregrinación: dos caras de la misma moneda en el camino português interior de santiago de compostela". Cuadernos del Turismo, [no prelo].

Pereiro Pérez, X. \& Fernandes, F. (2018): Antropologia e Turismo: teorias, métodos e praxis. Tenerife: PASOS, RTPC.

Remoaldo, P. \& Cadima Ribeiro, J. (2017): O legado de Guimarães Capital Europeia da Cultura de 2012: A leitura dos residentes e dos visitantes. Porto: Afrontamento.

Richards, G. (2014): Tourism trends: The convergence of culture and tourism [More Info: This paper is an update of Tourism trends: Tourism, culture and cultural routes (2011)].

Rodríguez Fernández, Ma . M. (2013): "La planificación y creación del evento Xacobeo: análisis de la identidad y la imagen", in Simone Novello et al (eds.). Xacobeo de un recurso a un evento turístico global, Santiago de Compostela, Andavira, pp. 65-87.

Rodríguez González, M. A. (2015): "Del espectáculo cultural y sus efectos: arte y políticas culturales en Santiago de Compostela". Espacio, tiempo y forma, serie VII, 3, 377-402.

Rodríguez Morató, A. (2005): La reinvención de la política cultural a escala local: el caso de Barcelona. Sociedade e Estado, vol. 20, 2, 351-377.

Rodríguez Morató, A. (2010): A metamorfose do valor cultural na sociedade contemporânea: desafios e paradoxos", in Santos M. L. \& Pais, J. (orgs.). Novos Trilhos Culturais. Práticas e Políticas Lisboa, Instituto de Ciências Sociais da Universidade de Lisboa, pp. 37-50.

Rodríguez Prado, M. F. (2015): “Textos ficcionais e práticas culturais de portugueses com relação a Santiago de Compostela: contrastes e homologias”, in Torres Feijó, BelloVázquez, Samartim \& Brito-Semedo (eds.), Estudos da AIL em Teoria e Metodologia Relacionamento nas Lusofonias I, Santiago de Compostela/Coimbra, Associação Internacional de Lusitanistas. pp. 143-157.

Santos Solla, X. M. (1999): "Mitos y realidades del Xacobeo", Boletín de la AGE, 27, 103-117. 
Santos Solla, X. M. (2006): "El camino de santiago: Turistas y peregrinos hacia Compostela”, Cuadernos de Turismo, núm. 18, pp. 135-150.

Santos Solla, X. M. \& Trillo-Santamaría, J. M. (2017): "Tourism and nation in Galicia (Spain)", Tourism Management Perspectives, 22: 98-108.

Scalabrini, E., Remaoldo, P., \& Lourenço, J. M. (2014): "Perceções de residentes a respeito dos impactes da atividade turística: Uma análise das publicações brasileiras sobre o tema", Tourism and Hospitality International Journal, 2(2), 12-31.

Simone Novello et al. (eds.) (2013): Xacobeo de un recurso a un evento turístico global, Santiago de Compostela: Andavira.

Somoza Medina, X. \& Lois González, R. C. (2017): “Ordenación del Territorio y estrategias de planificación en los Caminos de Santiago Patrimonio Mundial". Investigaciones Geográficas, (68), 47-63. https://doi.org/10.14198/INGEO2017.68.03 (28/06/2018).

Teixeira Coelho (1997): Dicionário crítico de política cultural. Cultura e Imaginário. São Paulo, Editora Iluminuras. http://hugoribeiro.com.br/biblioteca-digital/CoelhoDicionario_critico_de_politica_cultural.pdf (28/06/2018).

Torres Feijó, E. J. (2011): “Discursos contemporâneos e práticas culturais dominantes sobre Santiago e o Caminho: a invisibilidade da cultura como hipótese", in Lourenço A. A. \& Silvestre, O, M. (coords.), Literatura, espaço, cartografias, Coimbra, Centro de Literatura Portuguesa/Faculdade de Letras da Universidade de Coimbra, pp. 391-449.

Torres Feijó, E. J. (2012): "Interesses culturais e âmbitos receptivos em dous romances sobre o Caminho de Santiago: Frechas de Ouro e O Enigma de Compostela", Romance Notes, vol. 52, n2, 135-149.

Torres Feijó, E. J. (2014): “Autor, Texto e Espaço Geo-cultural Mediatizado. Processos de Ressemantização, Banalização e Misturação de Géneros em Produtos sobre o Caminho de Santiago", in Quintero, S. \& Baleiro, R. (eds.), Lit \& Tour: ensaios sobre literatura e turismo, Lisboa, Húmus, pp. 291-310.

Torres Feijó, E. J. (2015): "Identity Sustainability, Identity Affectivity, and the Ithaca Traveler: Conceptual Tools for Measuring and Modeling Tourism as an Opportunity", in Ricci, G. R. (ed.), Travel, Tourism and Identity, Culture \& Civilization, Volume 7, New Brunswick/ New Jersey, Transaction Publishers, pp. 143-162.

Turismo do Porto e Norte de Portugal (2015): Estratégia de Marketing Turístico do Porto e Norte de Portugal Horizonte 2015-2020, http://www.portoenorte.pt/fotos/gca/plano_estrategico_10327505915894b4d3a978b.pdf (28/06/2018).

UNESCO (s.d. [1985]): Santiago de Compostela (Old Town) [description]. http://whc. unesco.org/en/list/347/ (28/06/2018).

UNESCO (1998): Plan de Acción de Políticas Culturales para el Desarrollo http://unesdoc.unesco.org/images/0011/001163/116393So.pdf (28/06/2018).

Villares, R. (2017): Identidade e afectos patrios. Vigo, Galaxia. 